\title{
Numerical Modeling of Micron-Scale Flows Using the Gaussian Moment Closure
}

\author{
J. G. MDonald* and C. P. T. Groth ${ }^{\dagger}$ \\ University of Toronto Institute for Aerospace Studies \\ 4925 Dufferin Street, Toronto, Ontario, Canada, M3H 5 T6
}

\begin{abstract}
The application of the Gaussian moment closure to micron-scale flows is considered. The mathematical formulation of the closure is reviewed as well as an extension to allow for diatomic gases and treatment for solid wall boundaries. A parallel upwind finite-volume scheme with adaptive mesh refinement (AMR) using Roe and HLLE-type flux functions is described for solving the hyperbolic system of partial differential equations arising from this closure. Comparisons are made between numerical solutions of the Gaussian model and analytical solutions for several test problems, including Couette, boundary layer and cylinder flow, over a range of Knudsen numbers. Agreement between analytical and numeric solutions for these problems are very encouraging.
\end{abstract}

\section{Introduction}

Non-equilibrium micron-scale flows, such as those encountered in the complex micron-sized conduits of micro-electromechanical systems (MEMS) and flows associated with chemical-vapor deposition (CVD) processes commonly encountered in the manufacturing of semiconductor devices, $\frac{112]}{2}$ are difficult to solve using existing mathematical models and numerical methods. In most cases, these micron-scale flows are laminar and in the subsonic to low supersonic regimes having Mach numbers, Ma, less than two and low Reynolds numbers, Re. However, due to their micro-geometries, the characteristic lengths, $\ell$, of such flows are generally small and vary from 0.1 to $100 \mu \mathrm{m}$. Flow Knudsen numbers, $\mathrm{Kn}=\lambda / \ell$ where $\lambda$ is the particle mean free path, in the range $0.01<\mathrm{Kn}<10$ may be encountered, even for pressures above one atmosphere, and, as a result, non-continuum and thermal non-equilibrium effects can significantly influence momentum and heat transfer phenomena in typical micro-channel flows. ${ }^{3] 4 \sqrt[5]{6}}$ In many cases, these effects are not well understood and suitable mathematical theory and economical computational tools do not exist for attacking such problems. Although particle-simulation techniques, such as the direct-simulation Monte Carlo (DSMC) method of Bird, $\stackrel{7}{7}$ have been developed for for the prediction of general non-equilibrium gaseous flows, for slip and transitional regime flows (i.e., for $0.01<\mathrm{Kn}<1$ ) and for flows with low Mach numbers, the computational expenses incurred by these techniques are considerable and can currently prohibit their general usage. A discussion of the numerical difficulties associated with DSMC simulations for low-Mach-number and near-equilibrium flows is given by Breuer et al ${ }^{[}$New numerical methods capable of accurately and efficiently predicting non-equilibrium micro-scale flows in the transitional flow regime from the continuum flow to the near free-molecular flow regime would therefore be of practical importance to the fields of micromechanics and CVD process modeling and would greatly aid in the design and development of sophisticated micro-mechanical devices and improved semiconductor manufacturing techniques.

In recent studies, Levermor ${ }^{99}$ has proposed a new hierarchy of non-perturbative moment closures with several desirable mathematical properties. These methods are based on approximate solutions to the Boltzmann equation of kinetic theory and provide a hyperbolic mathematical description of non-equilibrium flows. Perturbative variants of these closures have subsequently been formulated by Groth et al $\frac{10] 11}{11}$ with a view to achieving practical and reliable mathematical tools for computation. The latter form a hierarchy of Gaussian-based perturbative moment closures which preliminary numerical studies by Brown et al $\frac{12 \sqrt[13]{13}}{1}$

*PhD Candidate, Email: mcdonald@utias.utoronto.ca, Student Member AIAA.

†Associate Professor, Email: groth@utias.utoronto.ca, Senior Member AIAA. 
indicate hold considerable promise for describing non-equilibrium transport, at least for subsonic and transonic flows in the slip and transition regimes. Aspects of the non-equilibrium solutions provided by the lowest order closure of both the Levermore non-perturbative and Gaussian-based perturbative hierarchies, the 10-moment or Gaussian closure, have been investigated by Levermore and Morokoff, 14 Brown et al., 12 Hittinger, $\frac{15}{15}$ and Suzuki and van Leer ${ }^{16}$ As in Grad-type closures, ${ }^{17}$ these closure models assume that, along with the density, flow velocity, and pressure, the viscous stresses, heat fluxes, and other higher-order moments of the velocity distribution are unknowns and the closures furnish transport equations for an extended set of macroscopic quantities. Consequently, the number of dependent variables can be large. Nevertheless, unlike the Navier-Stokes and Burnett models, which follow from Chapman-Enskog-type perturbative expansion techniques $\frac{18[19}{20}$ and have an elliptic nature, and previous Grad-type closures, that can breakdown and become ill-posed, the resulting set of quasi-linear moment equations remain stable and strictly hyperbolic, and their predicted moments realizable, such that closure breakdown is avoided for a considerable range of flow conditions 11

In addition to being mathematically well posed, the ability to treat non-equilibrium flows with a purely hyperbolic model, with no requirement to evaluate higher than first-order derivatives, is not only physically pleasing ${ }^{a}$ but may prove to be highly advantageous from a computational perspective. The hyperbolic moment equations of the closures described above seem very amenable to solution by the sophisticated nonlinear discretization procedures developed for the equations of continuum hydrodynamics, such as the various forms of Godunov-type finite-volume schemes.21|22|23|24|25 Schemes of this type are robust, minimize numerical discretization errors, provide accurate resolution of discontinuities, and permit the systematic application of physically realistic boundary conditions. Furthermore, the methods readily enable the application of unstructured adaptive mesh refinement (AMR) techniques for the treatment of complex geometries and for resolving highly disparate length scales while at the same time optimizing the usage of computational resources. $26,27,28$ They also have narrow stencils, making them suitable for implementation on high-speed massively parallel computer architectures.29 30 31

In the present work, the application of the Gaussian moment closure to micron-scale flows is considered. In particular, its solutions are explored using a parallel AMR Godunov-type finite-volume scheme. The mathematical theory and properties of the Gaussian closure for two-dimensional flows are reviewed and then the proposed parallel solution algorithm is discussed. The remainder of the paper is concerned with an assessment of the Gaussian closure for micron-scale flows. Numerical results are presented for several fundamental flow problems, including planar Couette, flat-plate boundary-layer flows and flow past a circular cylinder. The computed solutions of the Gaussian closure are compared with other analytical, experimental, and/or numerical results where possible. Although the Gaussian closure is deficient in terms of its description of thermal transport, it possesses many of the important features of more complete descriptions based on higher-order moment methods and this study represents the necessary first steps towards demonstrating the solvability of Gaussian-based closures and exploring the potential of models of this type to predict practical micron-scale flows.

\section{Governing Equations}

\section{A. Gaskinetic Theory}

In order to effectively treat non-continuum flows, the particle nature of fluids must be considered. Classical gaskinetic theory uses a probabilistic treatment to model particle behaviour. Probability density functions for the gas, $\mathcal{F}\left(x_{i}, v_{i}, t\right)$, are defined in six-dimensional phase space and specify the probability of finding particles at a given location, $x_{i}$, and time, $t$, having a particular gas velocity, $v_{i}$. Macroscopic "observable" properties of the gas are then obtained by taking appropriate velocity moments of $\mathcal{F}$. The evolution of the velocity distribution function is given by the Boltzmann equation. 32,33 This is an integro-differential equation for $\mathcal{F}$ having the form:

$$
\frac{\partial \mathcal{F}}{\partial t}+v_{i} \frac{\partial \mathcal{F}}{\partial x_{i}}+a_{i} \frac{\partial \mathcal{F}}{\partial v_{i}}=\frac{\delta \mathcal{F}}{\delta t}
$$

Here $a_{i}$ is the acceleration due to external forces and is taken to be zero for the present work. The term on the right hand side of the equation, $\frac{\delta \mathcal{F}}{\delta t}$, is the Boltzmann collision operator representing the time rate of change of the distribution function produced by inter-particle collisions. Mathematical models of the collision operator

\footnotetext{
${ }^{a}$ Hyperbolicity of the moment equations ensures finite speeds of propagation for infinitesimal disturbances.
} 
are in general very complex. The uncertainties associated with intermolecular force modeling and the detailed evaluation of complicated Boltzmann collision integrals can, however, be avoided by utilizing the relaxationtime or BGK approximation, as first proposed by Bhatnagar et al ${ }^{[35}$ In this simplified mathematical model, the collision operator is approximated by

$$
\frac{\delta \mathcal{F}}{\delta t}=-\frac{\mathcal{F}-\mathcal{M}}{\tau}
$$

where $\mathcal{M}$ is the equilibrium Maxwell-Boltzmann distribution towards which the solution is relaxing and $\tau$ is a characteristic relaxation time for the collision processes. The BGK operator preserves the usual collisional invariants and, under equilibrium conditions, $\delta F / \delta t=0$ and $F=\mathcal{M}$ as required. Note that the relaxation time model is only an approximation to the Boltzmann collision integral and ignores the detailed nature of inter-particle interactions. Nevertheless, it retains many of the qualitative features of the true collision integral and is thought to be sufficient for this study of micron-scale flows.

Transport equations governing the time evolution of macroscopic quantities can be derived by evaluating velocity moments of the Boltzmann equation given above. Letting

$$
\left\langle M\left(v_{i}\right) \mathcal{F}\right\rangle=\int_{-\infty}^{\infty} M\left(v_{i}\right) F\left(\left(x_{i}, v_{i}, t\right) d^{3} v,\right.
$$

then the so-called Maxwell's equation of change describing the transport of the moment $\langle M \mathcal{F}\rangle$ is given by

$$
\frac{\partial}{\partial t}\langle M \mathcal{F}\rangle+\frac{\partial}{\partial x_{i}}\left\langle v_{i} M \mathcal{F}\right\rangle=-\frac{1}{\tau}[\langle M \mathcal{F}\rangle-\langle M \mathcal{M}\rangle]
$$

where acceleration field is taken to be zero, the BGK relaxation time model has been used to approximate the collision operator, and $M$ is an appropriate velocity dependent weight.

\section{B. The 10-Moment Gaussian Model}

In moment closures of the type first proposed by Grad, 17 approximate solutions to the Boltzmann equation are constructed by assuming a particular form for the distribution function, $\mathcal{F}$, in terms of a finite number of free parameters or coefficients. The coefficients describing the approximate distribution function are then related to a finite number of velocity moments, the time evolution of which are given by Maxwell's equation of change. This procedure leads to a set of balance equations for the mass density, flow velocity, pressure, viscous stresses, heat fluxes, and other higher-order moments of the velocity distribution function. Closure of the transport equations is provided by expressing the higher-order moments as a function of the lower-order velocity moments as dictated by the approximate form for the distribution function.

In the Gaussian closure, the velocity distribution function for monatomic gaseous particles is assumed to have the following form:

$$
\mathcal{G}\left(x_{i}, v_{i}, t\right)=\frac{\rho}{m(2 \pi)^{3 / 2}(\operatorname{det} \boldsymbol{\Theta})^{1 / 2}} \exp \left(-\frac{1}{2} \Theta_{i j}^{-1} c_{i} c_{j}\right)
$$

where $m$ is the particle mass, $\rho$ is the mass density, $c_{i}$ is the random component of particle velocity given by $\mathbf{c}=\mathbf{v}-\mathbf{u}, \mathbf{u}$ is the average or bulk velocity of the particles, $\Theta_{i j}$ is a symmetric 'temperature' tensor given by $\Theta_{i j}=P_{i j} / \rho$ where $P_{i j}$ is the generalized pressure tensor. The fluid or deviatoric stress tensor, $\tau_{i j}$, is related to the pressure tensor as follows: $\tau_{i j}=\delta_{i j} p-P_{i j}$, where $p=P_{i i} / 3$ is the equilibrium isotropic pressure and $\tau_{k k}=0$ for a monatomic gas.

The Gaussian distribution appears to have been first derived in early work by Maxwell $\frac{36}{36}$ and then re-discovered in subsequent but independent research by both Schlüter ${ }^{37 \mid 38}$ and Holway! 39,40,41 It may be regarded as a generalization of the bi- and tri-Maxwellian velocity distribution functions that does not require the identification of the planes of principal stress. $37 \sqrt[38]{42} 43$ This approximate non-equilibrium distribution possesses a Gaussian-like distribution in each of the principal strain axes, physically, this corresponds to a non-equilibrium gas with a different temperature in each direction. Levermore ${ }^{9}$ has shown that for nonnegative density, $\operatorname{det} \boldsymbol{\Theta}>0$ and $\boldsymbol{\Theta}$ remain positive definite. Hence, $\boldsymbol{\Theta}^{-1}$ is also positive definite and $\Theta_{i j}^{-1} c_{i} c_{j}>0$ for all $\mathbf{c}$ other than $c_{i}=0$, from which it readily follows that $\mathcal{G}$ is finite and positive valued for 
all physically realistic values of $\rho$ and $\mathbf{P}$. Note that for flows in local thermal equilibrium, $P_{i j}=\delta_{i j} p$ and the Gaussian reduces to Maxwellian distribution as required (i.e., $\mathcal{G}=\mathcal{M}$ ).

The moment equations corresponding to the Gaussian closure can be obtained by substituting the weights $M=\left\{m, m v_{i}, m v_{i} v_{j}\right\}$ into Maxwell's equation of change, Eq. (4). This yields a set of ten partial-differential equations (PDEs) describing the transport of the macroscopic quantities $\rho, u_{i}$, and $P_{i j}$ which may be expressed as

$$
\begin{gathered}
\frac{\partial \rho}{\partial t}+\frac{\partial \rho u_{k}}{\partial x_{k}}=0 \\
\frac{\partial}{\partial t}\left(\rho u_{i}\right)+\frac{\partial}{\partial x_{k}}\left(\rho u_{i} u_{k}+P_{i k}\right)=0 \\
\frac{\partial P_{i j}}{\partial t}+\frac{\partial}{\partial x_{k}}\left(u_{k} P_{i j}\right)+P_{j k} \frac{\partial u_{i}}{\partial x_{k}}+P_{i k} \frac{\partial u_{j}}{\partial x_{k}}=-\frac{1}{\tau}\left(P_{i j}-\frac{1}{3} P_{k k} \delta_{i j}\right) .
\end{gathered}
$$

Note that by construction the third-order velocity moments of the Gaussian are zero, $\left\langle m c_{i} c_{j} c_{k} \mathcal{G}>=0\right.$, such that the heat flux vector, $q_{i}=\frac{1}{2}<m c_{i} c_{j} c_{j} \mathcal{G}>$ also vanishes. This points to a significant limitation of the Gaussian closure: its inability to account for the effects of thermal diffusion. Note also that when the solution reaches equilibrium and $\mathcal{G}=\mathcal{M}$, the Gaussian moment equations reduce to the Euler equations of equilibrium gas dynamics. Solution of Eqs. (6) -(8), subject to appropriate initial and boundary data, provides a complete description of the Gaussian velocity distribution function throughout the flow field of interest.

\section{An Extension for Diatomic Gases}

The transport equations of the Gaussian closure, Eqs. (6)-(8), were derived for a monatomic gas with three translational degrees of freedom and no rotational or vibrational degrees of freedom. The closure is therefore not immediately applicable to diatomic gases. A modification must be made to account for energy that can be present due to the extra internal degrees of freedom of diatomic molecules. The approach described by Hittinger $\sqrt{15}$ is used here for these purposes.

Under normal pressures and temperatures, vibrational degrees of freedom are usually not excited. It is therefore sufficient to simply model the translational and rotational degrees of freedom present in diatomic molecules. The energy associated with the translation of the centre of mass of a diatomic molecule will be equal to that of a monatomic molecule of equal weight. Therefore, the only addition required to the 10-moment Gaussian closure is a treatment of the energy present in the rotation of molecules. To do this, a "dumbbell" model of a diatomic molecule will be assumed. This model molecule is free to rotate about three different axes. Rotation about an axis that is aligned with the vector connecting the centers of mass of the two atoms will be considered to contain no energy, as the moment of inertia about this axis is negligible.

Internal energy can be present in the form of rotation about the remaining two axes. This energy will given by:

$$
\epsilon_{\text {rotation }}=\frac{1}{2} I_{\zeta} \omega_{\zeta}^{2}+\frac{1}{2} I_{\eta} \omega_{\eta}^{2}
$$

where $\zeta$ and $\eta$ are the two axis about which rotational energy may be present. Due to symmetry, $I_{\zeta}=I_{\eta}=I$ and therefore,

$$
\epsilon_{\text {rotation }}=\frac{1}{2} I\left(\omega_{\zeta}^{2}+\omega_{\eta}^{2}\right)
$$

By assuming that rotational velocities are statistically independent of each other and of the translational velocities, a modified Gaussian distribution for a diatomic gas can be written as:

$$
\mathcal{G}_{D}\left(x_{i}, v_{i}, \omega, t\right)=\mathcal{G}\left(x_{i}, v_{i}, t\right) g\left(x_{i}, \omega_{\zeta}, \omega_{\eta}, t\right)=\mathcal{G}\left(x_{i}, v_{i}, t\right) g_{\zeta}\left(x_{i}, \omega_{\zeta}, t\right) g_{\eta}\left(x_{i}, \omega_{\eta}, t\right)
$$

Furthermore, due to rotational symmetry, the angular velocity distribution may be expressed solely as a function of $x, t$ and $\omega$, where $\omega^{2}=\omega_{\zeta}^{2}+\omega_{\eta}^{2}$. By using the method of undetermined coefficients, this distribution can be shown to have the form:

$$
g\left(x_{i}, \omega, t\right)=g_{\zeta}\left(x_{i}, \omega, t\right) g_{\eta}\left(x_{i}, \omega, t\right)=\frac{B}{\pi} e^{\left(-B \omega^{2}\right)} .
$$


Then by making an analogy to the translational degrees of freedom, a rotational temperature can be defined as $T_{\text {rot }}=I / 2 \kappa B$. The distribution, $g$, then has the form

$$
g\left(x_{i}, \omega, t\right)=\left(\frac{I}{2 \pi \kappa T_{\text {rot }}}\right) e^{\left(\frac{-I}{2 \kappa T_{\text {rot }}} \omega^{2}\right)}=\left(\frac{I}{2 \pi \kappa T_{\text {rot }}}\right) e^{\left(-\frac{1}{2} R_{\alpha \beta} \omega_{\alpha} \omega_{\beta}\right)},
$$

where the tensor, $R_{\alpha \beta}$, is given by

$$
R_{\alpha \beta}=\frac{I}{\kappa T_{\text {rot }}} \delta_{\alpha \beta}=\frac{n I}{p}\left(\frac{T}{T_{\text {rot }}}\right) \delta_{\alpha \beta},
$$

and where $p=n k T=\rho R T$. The total velocity distribution is therefore:

$$
\mathcal{G}_{D}\left(x_{i}, v_{i}, \omega_{\zeta}, \omega_{\eta}, t\right)=\frac{\rho^{2} I}{m^{2}(2 \pi)^{5 / 2}(\operatorname{det} \Theta)^{1 / 2} p}\left(\frac{T}{T_{\text {rot }}}\right) \exp \left(-\frac{1}{2} \Theta_{i j}^{-1} c_{i} c_{j}\right) \exp \left(-\frac{1}{2} R_{\alpha \beta} \omega_{\alpha} \omega_{\beta}\right) .
$$

The Gaussian distribution function for a diatomic gas, $\mathcal{G}_{D}$, is defined for an 8-dimensional phase space consisting of three position coordinates, three translational velocity coordinates, and two angular velocity coordinates. For general non-equilibrium flows, the rotational and translational temperatures, $T$ and $T_{\text {rot }}$, need not be equal, they will however become equal when the gas is in thermodynamic equilibrium. In equilibrium, the distribution function, $\mathcal{G}_{D}$, for a diatomic gas becomes:

$$
\mathcal{M}_{D}\left(x_{i}, v_{i}, \omega_{\zeta}, \omega_{\eta}, t\right)=\frac{\rho I}{m^{2}(2 \pi p / \rho)^{5 / 2}} \exp \left(-\frac{1}{2} \frac{\rho}{p}\left[v^{2}+\frac{I}{m} \omega^{2}\right]\right),
$$

where $p$ is again the equilibrium pressure. For a diatomic gas, $p$ is still related to the generalized pressure tensor by $P_{i j}=p \delta_{i j}-\tau_{i j}$ and $P_{k k}=3 p-\tau_{k k}$ but now $\tau_{k k} \neq 0$. In fact, the normal deviatoric stresses are a measure of the departure of the rotational energy, $E_{\text {rot }}=p\left(T_{\text {rot }} / T\right)$ from its equilibrium value of $E_{\text {rot }}=p$.

Substituting Eq. (15) in to Maxwell's equation of change, Eq. (44), with weights $M=\left\{m, m v_{i}, m v_{i} v_{j}, I \omega^{2} / 2\right\}$ the following system of moment equations may be obtained:

$$
\begin{gathered}
\frac{\partial \rho}{\partial t}+\frac{\partial \rho u_{k}}{\partial x_{k}}=\left\langle m \frac{\delta \mathcal{G}_{D}}{\delta t}\right\rangle \\
\frac{\partial}{\partial t}\left(\rho u_{i}\right)+\frac{\partial}{\partial x_{k}}\left(\rho u_{i} u_{k}+P_{i k}\right)=\left\langle m v_{i} \frac{\delta \mathcal{G}_{D}}{\delta t}\right\rangle \\
\frac{\partial P_{i j}}{\partial t}+\frac{\partial}{\partial x_{k}}\left(u_{k} P_{i j}\right)+P_{j k} \frac{\partial u_{i}}{\partial x_{k}}+P_{i k} \frac{\partial u_{j}}{\partial x_{k}}=\left\langle m c_{i} c_{j} \frac{\delta \mathcal{G}_{D}}{\delta t}\right\rangle, \\
\frac{\partial E_{r o t}}{\partial t}+\frac{\partial u_{k} E_{r o t}}{\partial x_{k}}=\left\langle\frac{I \omega^{2}}{2} \frac{\delta \mathcal{G}_{D}}{\delta t}\right\rangle .
\end{gathered}
$$

This system of 11 nonlinear PDEs includes an additional scalar transport equation for the rotational energy, $E_{\text {rot }}=p\left(T_{\text {rot }} / T\right)$, and its solution provides a full prescription of the Gaussian distribution, $\mathcal{G}_{D}$.

To incorporate the effects of particle collisions, the BGK relaxation time model of Eq. (2) can be modified to account for the added degrees of freedom. The relaxation to equilibrium is now treated as a "two step" process where

$$
\frac{\delta \mathcal{G}_{D}}{\delta t}=-\frac{\mathcal{G}_{D}-\mathcal{F}_{D}}{\tau_{t}}-\frac{\mathcal{F}_{D}-\mathcal{M}_{D}}{\tau_{r}}
$$

In the diatomic case, it is assumed that the non-equilibrium distribution, $\mathcal{G}_{D}$, will relax toward a distribution, $\mathcal{F}_{D}$, where the translational degrees of freedom are in equilibrium with each other, but not in equilibrium with the rotational degrees of freedom. This relaxation will happen on a time scale $\tau_{t}$. The gas will then relax from there to the equilibrium distribution, $\mathcal{M}_{D}$, on a time scale $\tau_{r}$. Using this collision operator, the moment equations for the Gaussian closure can be re-written as:

$$
\begin{gathered}
\frac{\partial \rho}{\partial t}+\frac{\partial \rho u_{k}}{\partial x_{k}}=0 \\
\frac{\partial}{\partial t}\left(\rho u_{i}\right)+\frac{\partial}{\partial x_{k}}\left(\rho u_{i} u_{k}+P_{i k}\right)=0
\end{gathered}
$$

$$
5 \text { of } 19
$$




$$
\begin{gathered}
\frac{\partial P_{i j}}{\partial t}+\frac{\partial}{\partial x_{k}}\left(u_{k} P_{i j}\right)+P_{j k} \frac{\partial u_{i}}{\partial x_{k}}+P_{i k} \frac{\partial u_{j}}{\partial x_{k}}=-\frac{3 P_{i j}-P_{k k} \delta_{i j}}{3 \tau_{t}}-\frac{2\left(P_{k k}-3 E_{r o t}\right)}{15 \tau_{r}} \delta_{i j} \\
\frac{\partial}{\partial t}\left(E_{r o t}\right)+\frac{\partial}{\partial x_{k}}\left(u_{k} E_{r o t}\right)=-\frac{3 E_{r o t}-P_{k k}}{5 \tau_{r}}
\end{gathered}
$$

Note that by combining the transport equations above, a conservation equation for the total energy of the gas can be obtained and written as

$$
\frac{\partial}{\partial t}\left(\frac{1}{2} \rho u^{2}+\frac{1}{2} P_{j j}+E_{r o t}\right)+\frac{\partial}{\partial x_{k}}\left(u k\left[\frac{1}{2} \rho u^{2}+\frac{1}{2} P_{j j}+E_{r o t}\right]+u_{j} P_{j k}\right)=-\frac{P_{k k}-3 p}{2 \tau_{r}}-\frac{E_{r o t}-p}{\tau_{r}}
$$

As the total energy must be conserved, the source terms associated with relaxation to equilibrium must balance each other and vanish. It is a simple matter to show that $\tau_{k k}=2\left(E_{\text {rot }}-p\right)$, clearly showing the relationship between the normal fluid stresses and deviation of the rotational energy from it equilibrium value. Note also that, in equilibrium, Eqs. (22)-(20) properly reduce to the Euler equations with one additional equation for the convection of rotational energy.

\section{Eigenstructure for Two-Dimensional Flows}

In two dimensional planar flows, Eqs. (22)-(25) can be written in weak conservation form as:

$$
\frac{\partial \mathbf{U}}{\partial t}+\frac{\partial \mathbf{F}}{\partial x}+\frac{\partial \mathbf{G}}{\partial y}=\mathbf{S}
$$

where $\mathbf{U}$ is the vector of conserved variables, and $\mathbf{F}$ and $\mathbf{G}$ are $x$ and $y$ direction flux vectors given by:

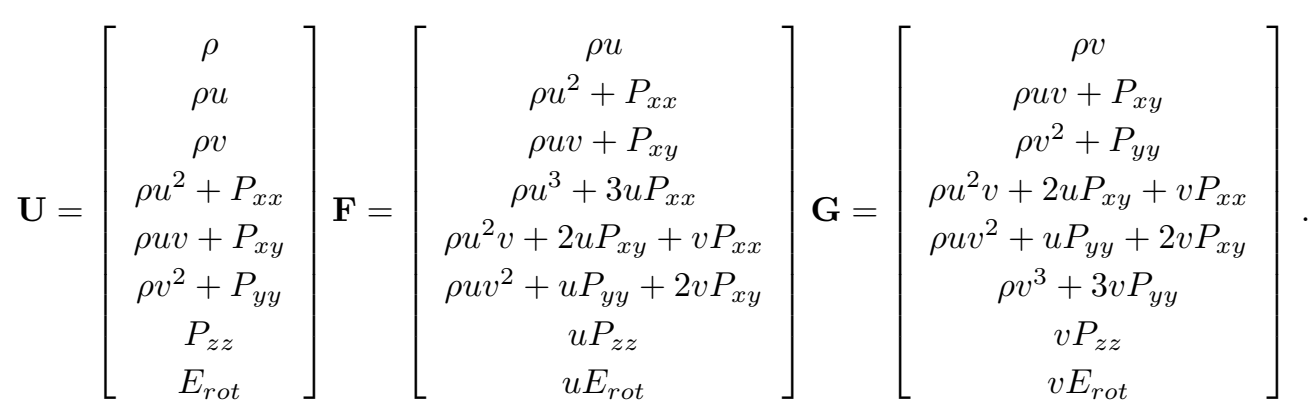

The source vector $\mathbf{S}$ of Eq. (27) has the form:

$$
\mathbf{S}=\left[\begin{array}{c}
0 \\
0 \\
0 \\
-\frac{1}{3 \tau_{t}}\left(2 P_{x x}-P_{y y}-P_{z z}\right)-\frac{2}{15 \tau_{r o t}}\left(P_{x x}+P_{y y}+P_{z z}-3 E_{r o t}\right) \\
-\frac{1}{\tau_{t}} P_{x y} \\
-\frac{1}{3 \tau_{t}}\left(2 P_{y y}-P_{x x}-P_{z z}\right)-\frac{2}{15 \tau_{r o t}}\left(P_{x x}+P_{y y}+P_{z z}-3 E_{r o t}\right) \\
-\frac{1}{3 \tau_{t}}\left(2 P_{z z}-P_{x x}-P_{y y}\right)-\frac{2}{15 \tau_{r o t}}\left(P_{x x}+P_{y y}+P_{z z}-3 E_{r o t}\right) \\
-\frac{1}{5 \tau_{r o t}}\left(3 E_{r o t}-P_{x x}-P_{y y}-P_{z z}\right)
\end{array}\right] .
$$

By making use of the flux Jacobians $\mathbf{A}=\frac{\partial \mathbf{F}}{\partial \mathbf{U}}$ and $\mathbf{B}=\frac{\partial \mathbf{G}}{\partial \mathbf{U}}$, Eq. (27) can be re-written as:

$$
\frac{\partial \mathbf{U}}{\partial t}+\mathbf{A} \frac{\partial \mathbf{U}}{\partial x}+\mathbf{B} \frac{\partial \mathbf{U}}{\partial y}=\mathbf{S}
$$

The source terms require the specification of two relaxation times. Approximate expressions are used here to relate the relaxation times to the gas viscosities. They have the following form:

$$
\tau_{t}=\frac{\mu}{p}, \quad \tau_{r}=\frac{15 \mu_{B}}{4 p}
$$


where $\mu$ is the fluid viscosity, $\mu_{B}$ is the bulk viscosity and $p$ is the thermodynamic pressure; empirical relations can be used to determine the related viscosities.

Levermore ${ }^{9}$ Brown et al. ${ }^{12[13]}$ and Hittinge ${ }^{15}$ have shown that the inhomogeneous PDEs given in Eq. (28) are strictly hyperbolic (i.e., the eigenvalues of $\mathbf{A}$ and $\mathbf{B}$ are all real and the corresponding eigenvectors are linearly independent and form a complete set). Physically, the eigenvalues of the flux Jacobians describe the characteristic speeds for the propagation of infinitesimal disturbances and the eigenvectors characterize the transport of the fluid quantities associated with these fundamental solution modes. The results of the previous eigenstructure studies are now briefly reviewed here, both to illustrate the strict hyperbolic nature of the Gaussian closure and in order to develop the numerical flux functions that are used in the proposed upwind-biased finite volume scheme to solve the governing equations of the Gaussian model.

For planar propagations in the $x$-direction, a characteristic analysis reveals that the eight eigenvalues, $\lambda_{k}$, of the $8 \times 8$ matrix $\mathbf{A}$ are the roots of the polynomial equation $\operatorname{det}(\mathbf{A}-\lambda \mathbf{I})$ and have are given by

$$
\lambda_{1-8}=\left(u-\sqrt{3} c_{x x}, u-c_{x x}, u, u, u, u, u+c_{x x}, u+\sqrt{3} c_{x x}\right),
$$

where $c_{x x}^{2} \rho=P_{x x}$. A complete set of linearly independent eigenvectors can also be defined. The eight right eigenvectors, $\mathbf{r}$, are column vectors satisfying $\mathbf{A r}=\lambda \mathbf{r}$ and may be written as

$$
\begin{aligned}
& \mathbf{r}_{c 1}=\left[\begin{array}{c}
1 \\
u-\sqrt{3} c_{x x} \\
v-\frac{\sqrt{3} P_{x y}}{c_{x x} \rho} \\
3 c_{x x}^{2}-2 \sqrt{3} u c_{x x}+u^{2} \\
\frac{u \rho v c_{x x}-u \sqrt{3} P_{x y}-\sqrt{3} c_{x x}^{2} \rho v+3 c_{x x} P_{x y}}{\rho_{x x} \rho} \\
\frac{\rho^{2} v^{2} c_{x x}^{2}-2 \sqrt{3} P_{x y} c_{x x} \rho+\rho c_{x x}^{2} P_{y y}+2 P_{x y}^{2}}{\rho^{2} c_{x x}^{2}} \\
\frac{P_{z z}}{\rho} \\
\frac{E_{r o t}}{\rho}
\end{array}\right], \quad \mathbf{r}_{c 2}=\left[\begin{array}{c}
0 \\
0 \\
1 \\
0 \\
u-c_{x x} \\
2\left(v-\frac{P_{x y}}{c_{x x} \rho}\right) \\
0 \\
0
\end{array}\right], \\
& \mathbf{r}_{c 3}=\left[\begin{array}{c}
1 \\
u \\
v \\
u^{2} \\
u v \\
v^{2} \\
0 \\
0
\end{array}\right], \mathbf{r}_{c 4}=\left[\begin{array}{l}
0 \\
0 \\
0 \\
0 \\
0 \\
1 \\
0 \\
0
\end{array}\right], \mathbf{r}_{c 5}=\left[\begin{array}{l}
0 \\
0 \\
0 \\
0 \\
0 \\
0 \\
1 \\
0
\end{array}\right], \mathbf{r}_{c 6}=\left[\begin{array}{l}
0 \\
0 \\
0 \\
0 \\
0 \\
0 \\
0 \\
1
\end{array}\right] \text {, } \\
& \mathbf{r}_{c 7}=\left[\begin{array}{c}
0 \\
0 \\
1 \\
0 \\
u+c_{x x} \\
2\left(v+\frac{P_{x y}}{c_{x x} \rho}\right) \\
0 \\
0
\end{array}\right], \mathbf{r}_{c 8}=\left[\begin{array}{c}
1 \\
u+\sqrt{3} c_{x x} \\
v+\frac{\sqrt{3} P_{x y}}{c_{x x} \rho} \\
3 c_{x x}^{2}+2 \sqrt{3} u c_{x x}+u^{2} \\
\frac{u \rho v c_{x x}+u \sqrt{3} P_{x y}+\sqrt{3} c_{x x}^{2} \rho v+3 c_{x x} P_{x y}}{c_{x x} \rho} \\
\frac{\rho^{2} v^{2} c_{x x}^{2}+2 \sqrt{3} P_{x y} c_{x x} \rho v+\rho c_{x x}^{2} P_{y y}+2 P_{x y}^{2}}{\rho^{2} c_{x x}^{2}} \\
\frac{P_{z z}}{\rho} \\
\frac{E_{r o t}}{\rho}
\end{array}\right] .
\end{aligned}
$$

Letting $\mathbf{W}=\left[\rho, u, v, P_{x x}, P_{x y}, P_{y y}, P_{z z}, E_{r o t}\right]^{\mathrm{T}}$ be the row vector of primitive solution vectors variables and $\mathbf{M}=\frac{\partial \mathbf{U}}{\partial \mathbf{W}}$ be the Jacobian relating these primitive and conserved variables, then the $8 \times 8$ matrix $\mathbf{R}_{p}$ whose columns are composed of the right eigenvectors for the primitive variables is given by $\mathbf{R}_{p}=\mathbf{M}^{-1} \mathbf{R}_{c}$, where $\mathbf{R}_{c}$ is the matrix whose columns are the eight right eigenvectors for conserved variables. The left eigenvectors, $\mathbf{l}$ (row vectors satisfying $\mathbf{l} \mathbf{A}=\lambda \mathbf{l}$ ) can then be determined by inverting $\mathbf{R}_{p}$ to arrive at the matrix of left 
eigenvectors for primitive variables, $\mathbf{L}_{p}=\mathbf{R}_{p}^{-1}$. The resulting left eigenvectors are:

$$
\begin{gathered}
\mathbf{l}_{p 1}=\left[0,-\frac{\rho \sqrt{3}}{6 c_{x x}}, 0, \frac{1}{6 c_{x x}^{2}}, 0,0,0,0\right], \quad \mathbf{l}_{p 2}=\left[0,-\frac{P_{x y}}{2 c_{x x}^{2}}, \frac{\rho}{2}, \frac{P_{y x}}{2 \rho c_{x x}^{3}},-\frac{1}{2 c_{x x}}, 0,0,0\right], \\
\mathbf{l}_{p 3}=\left[1,0,0,-\frac{1}{3 c_{x x}^{2}}, 0,0,0,0\right], \mathbf{l}_{p 4}=\left[0,0,0,-\frac{\rho c_{x x}^{2} P_{y y}-4 P_{x y}^{2}}{3 \rho^{2} c_{x x}^{4}},-\frac{2 P_{x y}}{c_{x x}^{2} \rho}, 1,0,0\right], \\
\mathbf{l}_{p 5}=\left[0,0,0,-\frac{P_{z z}}{3 c_{x x}^{2} \rho}, 0,0,1,0\right], \quad \mathbf{l}_{p 6}=\left[0,0,0,-\frac{E_{r o t}}{3 c_{x x}^{2} \rho}, 0,0,0,1\right], \\
\mathbf{l}_{p 7}=\left[0,-\frac{P_{x y}}{2 c_{x x}^{2}}, \frac{\rho}{2},-\frac{P_{y x}}{2 \rho c_{x x}^{3}}, \frac{1}{2 c_{x x}}, 0,0,0\right], \mathbf{l}_{p 8}=\left[0, \frac{\rho \sqrt{3}}{6 c_{x x}}, 0, \frac{1}{6 c_{x x}^{2}}, 0,0,0,0\right],
\end{gathered}
$$

It should be evident that the eigenvalues of $\mathbf{A}$ are real for $\rho>0$ and $P_{x x}>0$. Moreover, we have a complete set of linearly independent left and right eigenvectors. As the choice of the $x$-direction for performing the preceding analysis was arbitrary, the Gaussian closure are therefore strictly hyperbolic provided that $P_{x x} / \rho>0$ for any orientation of the Cartesian coordinate frame. This condition amounts to requiring that $\Theta$ be positive definite or, for two-dimensional flows, $\rho>0$ and $P_{z z}\left(P_{x x} P_{y y}-P_{x y}^{2}\right) / \rho^{3}>0$.

The waves associated with the $\lambda_{1}$ and $\lambda_{8}$ eigenvalues are acoustic waves and are truly non-linear (i.e., self-steeping waves that can lead to the formation of shocks). The waves associated with $\lambda_{2}$ and $\lambda_{7}$ are shear waves. Finally, the waves associated with $\lambda_{3}, \lambda_{4} \lambda_{5}$, and $\lambda_{6}$ eigenvalues contain an entropy wave, two transverse pressure waves, and a rotational energy wave. Brown et al ${ }^{[1]}$ and Hittinger ${ }^{[15}$ provide a more detailed discussion of the nature of the fundamental solution modes waves associated with the Gaussian closure, including dispersion analysis showing the relaxation of the system to the equilibrium Euler equations.

\section{E. Roe Average State}

Roe-averaged flux Jacobians ${ }^{44}$ of the conservation form of equations given in Eq. (28) are required in order to construct the upwind-biased numerical flux functions. A suitable Roe average state or linearization can be determined using the 'assumed-form' or 'corrected-average' approach described by Brown 13 and Brown et al ${ }^{12}$ Letting $W_{i j}=\frac{P_{i j}}{\rho}$, values of the primitive variables defining the Roe average state for the Gaussian closure can be shown to be:

$$
\begin{gathered}
\hat{\rho}=\sqrt{\rho_{L} \rho_{R}}, \quad \hat{u}_{i}=\frac{\sqrt{\rho_{R}} u_{i R}+\sqrt{\rho_{L}} u_{i L}}{\sqrt{\rho_{R}}+\sqrt{\rho_{L}}}, \\
\hat{W}_{i j}=\frac{\sqrt{\rho_{R}} W_{i j R}+\sqrt{\rho_{L}} W_{i j L}}{\sqrt{\rho_{R}}+\sqrt{\rho_{L}}}+\frac{1}{3} \frac{\sqrt{\rho_{L} \rho_{R}}}{\left(\sqrt{\rho_{R}}+\sqrt{\rho_{L}}\right)^{2}} \Delta u_{i} \Delta u_{j}, \quad \hat{E}_{r o t}=\frac{\sqrt{\rho_{R}} E_{r o t R}+\sqrt{\rho_{L}} E_{r o t L}}{\sqrt{\rho_{R}}+\sqrt{\rho_{L}}} .
\end{gathered}
$$

\section{F. Solid Wall Boundary Conditions}

Appropriate solid wall boundary conditions for the Gaussian closure are not immediately obvious. One technique for determining the solution at a wall is to assume that there exists a Knudsen layer next to the solid surface ${ }^{17}$ as illustrated in Figure 1. In this infinitesimally thin layer, the fluid exists as a combination of the distribution function defining incoming particles from the interior flow field and a distribution function defining reflected particles arising from the wall. For example, for a solid wall extending in the x-direction with a fluid above it, all the particles with negative y-direction velocities in the Knudsen layer will come from the neighboring fluid with statistical properties defined by the Gaussian. In order to model the particle interaction with the wall, an accommodation coefficient, $0 \leq \alpha \leq 1$, is then defined. If $\alpha$ is zero (specular reflection), the incoming particles will simply be reflected specularly from the wall back into the Knudsen layer. For $\alpha=1$ (diffuse reflection), incoming particles are fully accommodated and will therefore come into

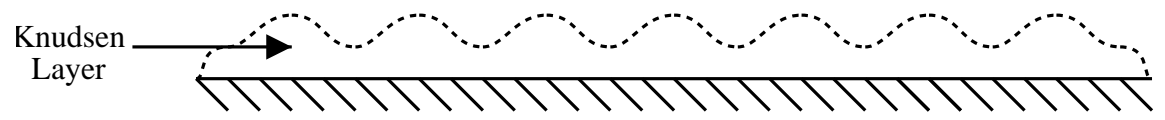

Figure 1. Knudsen layer at solid wall. 
thermodynamic equilibrium with the wall before being released from the wall and will re-enter the Knudsen layer with the statistical properties of a Maxwell-Boltzmann distribution defined by a wall temperature, $T_{w}$. For any intermediate $\alpha$ value, the reflected particles will enter the Knudsen layer as a combination of the two cases. The resulting distributions function for the Knudsen layer is then given by

$$
\mathcal{F}_{K n}=\mathcal{F}^{+}+\mathcal{F}^{-}
$$

where $\mathcal{F}^{+}$and $\mathcal{F}^{-}$are given by

$$
\begin{gathered}
\mathcal{F}^{-}=\left\{\begin{array}{cc}
\mathcal{G}_{b}\left(v_{x}, v_{y}, v_{z}\right) & \text { for } v_{y}<0, \\
0 & \text { for } v_{y}>0,
\end{array}\right. \\
\mathcal{F}^{+}=\left\{\begin{array}{cc}
\alpha \mathcal{M}_{w}\left(v_{x}, v_{y}, v_{z}\right)+(1-\alpha) \mathcal{G}_{b}\left(v_{x},-v_{y}, v_{z}\right) & \text { for } v_{y}>0, \\
0 & \text { for } v_{y}<0,
\end{array}\right.
\end{gathered}
$$

and where $\mathcal{G}_{b}$ is Gaussian distribution at the edge of the Knudsen layer and $\mathcal{M}_{w}$ is the Maxwellian defining particles which are fully accommodated by the wall. By assuming that the bulk y-direction velocity of the fluid immediately above the wall is zero and by imposing the constraint that the net particle flux through the wall must be zero, it is possible to show that the reflected Maxwellian has the form:

$$
\mathcal{M}_{w}\left(v_{x}, v_{y}, v_{z}\right)=\sqrt{\frac{n P_{y y}}{k T_{w}}}\left(\frac{m}{2 \pi k T_{w}}\right)^{\frac{3}{2}} e^{-\left(\frac{m}{2 k T_{w}}\right)\left(\left(v_{x}-u_{w x}\right)^{2}+v y^{2}+v z^{2}\right)},
$$

where all the properties are those of the fluid outside the Knudsen layer with the exception of $T_{w}$ which is the temperature of the wall and $u_{w x}$ which is the $\mathrm{x}$-direction velocity of the wall. A quick inspection of the first term in this distribution function finds that it relates the number density of the reflected Maxwellian to that of the incoming Gaussian distribution such that:

$$
\frac{n_{\text {Maxwellian }}}{n_{\text {Gaussian }}}=\sqrt{\frac{m P_{y y}}{\rho k T_{w}}},
$$

It can be shown that if the fluid interacts adiabatically with the wall, this ratio will be one, and Eq. (33) becomes:

$$
\mathcal{M}_{w}\left(v_{x}, v_{y}, v_{z}\right)=n\left(\frac{m}{2 \pi k T}\right)^{\frac{3}{2}} e^{-\left(\frac{m}{2 k T}\right)\left(\left(v_{x}-u_{w x}\right)^{2}+v y^{2}+v z^{2}\right)}
$$

where $T$ is now the fluid temperature.

Requiring the component of the bulk velocity of the fluid normal to solid walls to be zero is a very natural boundary condition for the Gaussian closure and provides one value for the required boundary data. The eigenvalues of the system, however, suggest that two boundary data are required to ensure that the problem is well-posed. Realizing that in the equilibrium limit, with no accommodation at the wall, the wall shear stress must be zero in order to recover the Euler equations, it seems that a boundary condition for $P_{x y}$ would be most appropriate. In order to find this condition, the appropriate velocity moment of $\mathcal{F}_{K n}$ must be evaluated. The bulk velocity of the Knudsen layer velocity distribution defined by Eq. (32) is also required as an intermediate step, this velocity however is not enforced as a boundary condition. It then follows that the following boundary conditions are appropriate for the Gaussian closure in the case of a solid wall extending in the x-direction:

$$
\begin{gathered}
u_{y}=0, \quad P_{x y}=\alpha\left[\frac{P_{x y}}{2}-\sqrt{\frac{\rho P_{y y}}{2 \pi}}\left(u_{x}-\bar{u}\right)+\sqrt{\frac{\rho_{w} n_{w} k T_{w}}{2 \pi}}\left(u_{w}-\bar{u}\right)\right], \\
\bar{u}=(2-\alpha)\left[\frac{u_{x}}{2}-\frac{P_{x y}}{\sqrt{2 \pi \rho P_{y y}}}\right]+\frac{\alpha}{2} \sqrt{\frac{P_{y y}}{n_{w} k T_{w}}} u_{w}
\end{gathered}
$$

with all properties being those of the incoming Gaussian distribution with the exception of: $\rho_{w}, n_{w}$ and $T_{w}$, which are defined by the Maxwellian reflected from the wall. It can easily be seen that this recovers the proper "no-shear" limit for specular reflection $(\alpha=0)$. If the wall is assumed to be adiabatic, Eq. (36) can be simplified as:

$$
u_{y}=0, \quad P_{x y}=\alpha\left[\frac{P_{x y}}{2}+\sqrt{\frac{\rho P_{y y}}{2 \pi}}\left(u_{w x}-u_{x}\right)\right],
$$

$$
9 \text { of } 19
$$


where all properties are those of the fluid, with the exception of $u_{w x}$, which is the velocity of the wall. Assuming no heat is transfered between the fluid and the wall is a natural assumption as the Gaussian closure does not allow heat transfer within the fluid. The simplified boundary conditions presented in Eq. (37) are used for all presented cases.

\section{Parallel AMR Finite-Volume Scheme}

\section{A. Finite Volume Method with Point-Implicit Time Marching}

A parallel higher-order Godunov-type finite-volume scheme has been developed to solve the transport equations of the Gaussian closure for two-dimensional planar flows on multi-block quadrilateral meshes. As the relaxation times, $\tau_{t}$ and $\tau_{r}$, can become very small in the near-equilibrium limit, a point-implicit time marching method is used to integrate the set of ordinary differential equations that result from the spatial discretization of the governing equations and deal with excessive numerical stiffness associated with the source terms. The fully-discrete finite-volume formulation with second-order semi-implicit time stepping applied to cell $(i, j)$ is given by

$$
\begin{gathered}
\tilde{\mathbf{U}}_{i, j}^{n+1}=\mathbf{U}_{i, j}^{n}-\frac{\Delta t}{A_{i, j}}\left(\sum_{k}(\overrightarrow{\mathbf{F}} \cdot \overrightarrow{\mathbf{n}} \Delta \ell)_{i, j, k}^{n}\right)+\Delta t \tilde{\mathbf{S}}_{i, j}^{n+1}, \\
\mathbf{U}_{i, j}^{n+1}=\mathbf{U}_{i, j}^{n}-\frac{\Delta t}{2 A_{i, j}}\left(\sum_{k}(\overrightarrow{\mathbf{F}} \cdot \overrightarrow{\mathbf{n}} \Delta \ell)_{i, j, k}^{n}+\sum_{k}(\tilde{\overrightarrow{\mathbf{F}}} \cdot \overrightarrow{\mathbf{n}} \Delta \ell)_{i, j, k}^{n+1}\right)+\Delta t\left(\frac{\mathbf{S}_{i, j}^{n}+\tilde{\mathbf{S}}_{i, j}^{n+1}}{2}\right),
\end{gathered}
$$

where $\mathbf{U}_{i, j}$ is the conserved state for cell $(i, j), \overrightarrow{\mathbf{F}}=(\mathbf{F}, \mathbf{G})$ is the flux dyad, $A_{i, j}$ is the area of the cell, $\Delta \ell$ and $\overrightarrow{\mathbf{n}}$ are the length of the cell face and unit vector normal to the cell face or edge, respectively, and the superscript $n$ is the index for the time step of size $\Delta t$. The numerical fluxes at the faces of each cell, $(\overrightarrow{\mathbf{F}} \cdot \overrightarrow{\mathbf{n}} \Delta \ell)_{i, j, k}$, are determined from the solution of a Riemann problem posed in a direction defined by the normal to the face. The left and right solution states for the Riemann problems are determined via a the least-squares piece-wise limited linear solution reconstruction procedure with either the Barth-Jesperson or Venkatakrishnan limiters. 45 [46 This provides a second-order accurate spatial discretization for smooth solutions. In the present algorithm, both Roe and HLLE-type approximate Riemann solvers $\underline{44}$ are used to solve the Riemann problem and evaluate the numerical flux.

If the relaxation times are assumed to be constant throughout the time step, the source terms can be rewritten as $\mathbf{S}^{n}=\mathbf{K}^{n} \mathbf{U}^{n}$ with

$$
\mathbf{K}=\left[\begin{array}{cccccccc}
0 & 0 & 0 & 0 & 0 & 0 & 0 & 0 \\
0 & 0 & 0 & 0 & 0 & 0 & 0 & 0 \\
0 & 0 & 0 & 0 & 0 & 0 & 0 & 0 \\
a u^{2}+b v^{2} & -2 a u & -2 b v & a & 0 & b & b & \frac{2}{5 \tau_{r}} \\
\frac{-u v}{\tau_{t}} & \frac{v}{\tau_{t}} & \frac{u}{\tau_{t}} & 0 & \frac{-1}{\tau_{t}} & 0 & 0 & 0 \\
b u^{2}+a v^{2} & -2 b u & -2 a v & b & 0 & a & b & \frac{2}{5 \tau_{r}} \\
b u^{2}+b v^{2} & -2 b u & -2 b v & b & 0 & b & a & \frac{2}{5 \tau_{r}} \\
\frac{u^{2}+v^{2}}{5 \tau_{r}} & \frac{-2 u}{5 \tau_{r}} & \frac{-2 v}{5 \tau_{r}} & \frac{1}{5 \tau_{r}} & 0 & \frac{1}{5 \tau_{r}} & \frac{1}{5 \tau_{r}} & \frac{-3}{5 \tau_{r}}
\end{array}\right]
$$

$a=-\frac{2}{3 \tau_{t}}-\frac{2}{15 \tau_{r}}$ and $b=\frac{1}{3 \tau_{t}}-\frac{2}{15 \tau_{r}}$. A matrix $\mathbf{B}$ can then be defined as: $\mathbf{B}=\mathbf{I}-\frac{\Delta t}{\Omega} \mathbf{K}$. Due to the local nature of the source terms, the matrix $\mathbf{B}$ can be inverted analytically and the preceding finite-volume time marching scheme can be rewritten as:

$$
\begin{gathered}
\tilde{\mathbf{U}}_{i, j}^{n+1}=\mathbf{B}_{i, j}^{-1}\left[\mathbf{U}_{i, j}^{n}-\frac{\Delta t}{A_{i, j}}\left(\sum_{k}(\overrightarrow{\mathbf{F}} \cdot \overrightarrow{\mathbf{n}} \Delta \ell)_{i, j, k}^{n}\right)\right], \\
\mathbf{U}_{i, j}^{n+1}=\mathbf{B}_{i, j}^{-1}\left[\mathbf{U}_{i, j}^{n}-\frac{\Delta t}{2 A_{i, j}}\left(\sum_{k}(\overrightarrow{\mathbf{F}} \cdot \overrightarrow{\mathbf{n}} \Delta \ell)_{i, j, k}^{n}+\sum_{k}(\tilde{\overrightarrow{\mathbf{F}}} \cdot \overrightarrow{\mathbf{n}} \Delta \ell)_{i, j, k}^{n+1}\right)+\frac{\Delta t}{2} \mathbf{S}_{i, j}^{n}\right],
\end{gathered}
$$

This time marching formulation allows the maximum time step to be determined by the usual CFL condition rather than being governed by the relaxation time scales, $\tau_{t}$ and $\tau_{r}$, of the stiff source terms. 


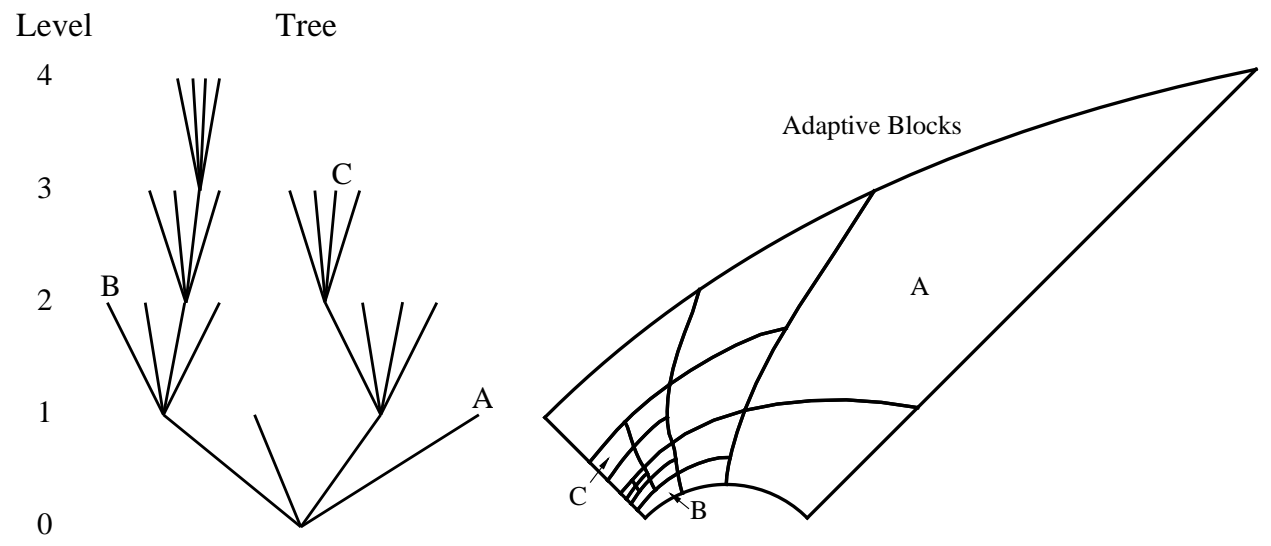

Figure 2. Quadtree data structure and block-based refinement for body-fitted mesh showing solution blocks at 4 levels of refinement.

\section{B. Parallel Adaptive Mesh Refinement}

Following the approach developed by Sachdev et al. ${ }^{48}$ the finite-volume scheme described above has been combined and implemented within a parallel block-based AMR solution procedure that enables automatic solution-directed mesh adaptation on body-fitted multi-block quadrilateral mesh and leads to an efficient and scalable parallel solution algorithm on distributed-memory multi-processor architectures. The proposed AMR formulation borrows from previous work by Berger and co-workers, $\frac{26] 49] 28}{20}$ Quirk $\frac{50[29}{29}$ and De Zeeuw and Powell ${ }^{27}$ for Cartesian mesh and has similarities with the block-based approaches described by Quirk and Hanebutte $\underset{29}{, 29}$ Berger and Saltzman, $\frac{49}{,}$ and Groth et al ${ }^{[30}$ Note that other researchers have considered the extension of Cartesian mesh adaptation procedures to more arbitrary quadrilateral and hexagonal mesh. See, for example, the work by Davis and Dannenhoffer $\underline{[51}$ and Sun and Takayama .52$]$

In the AMR scheme, an initial body-fitted multi-block mesh is automatically refined in areas of interest according to several physics-based refinement criteria. This is done by dividing a single "parent" block into four "child" blocks each with the same number of cells as the "parent" block; thereby doubling the grid resolution with each refinement. Overlapping ghost-cells are used to pass information from one block to another, making the block boundaries entirely transparent to the solution. The ghost cells are also used for the implementation of boundary conditions. The inter-connectivity of the solution blocks is stored in a tree-like data structure in which new branches are created at each refinement. This "quadtree" data structure allows for the connectivity of the blocks as well as the relative refinement levels to be stored as shown in Figure 2 Standard multigrid-type restriction and prolongation techniques are used when refining or coarsening blocks and flux corrections must be carried out at interfaces between blocks with different levels of refinement in order to ensure the scheme remains conservative.

The parallel AMR scheme is designed to be easily implemented on large multi-processor distributedmemory computing facilities. The similarity between blocks of all refinement levels ensures that they each require approximately the same computational effort to update the solution at each time step. Thus, parallel implementation is carried out via domain decomposition where the solution blocks are simply distributed equally among the available processors, with more than one block permitted on each processor. A Morton ordering space filling curve is used to provide nearest-neighbour ordering of blocks for efficient load balancing. ${ }^{31}$

\section{Parallel Performance}

Parallel implementation of the proposed algorithm has been carried out on parallel cluster of 4-way HewlettPackard ES40, ES45, and Integrity rx4640 servers with a total of 244 Alpha and Itanium 2 processors. A lowlatency Myrinet network and switch is used to interconnect the servers in the cluster. The implementation was carried out using the $\mathrm{C}++$ programming language and the MPI (message passing interface) library. Estimates of the parallel performance and scalability of the algorithm on this architecture are shown in 


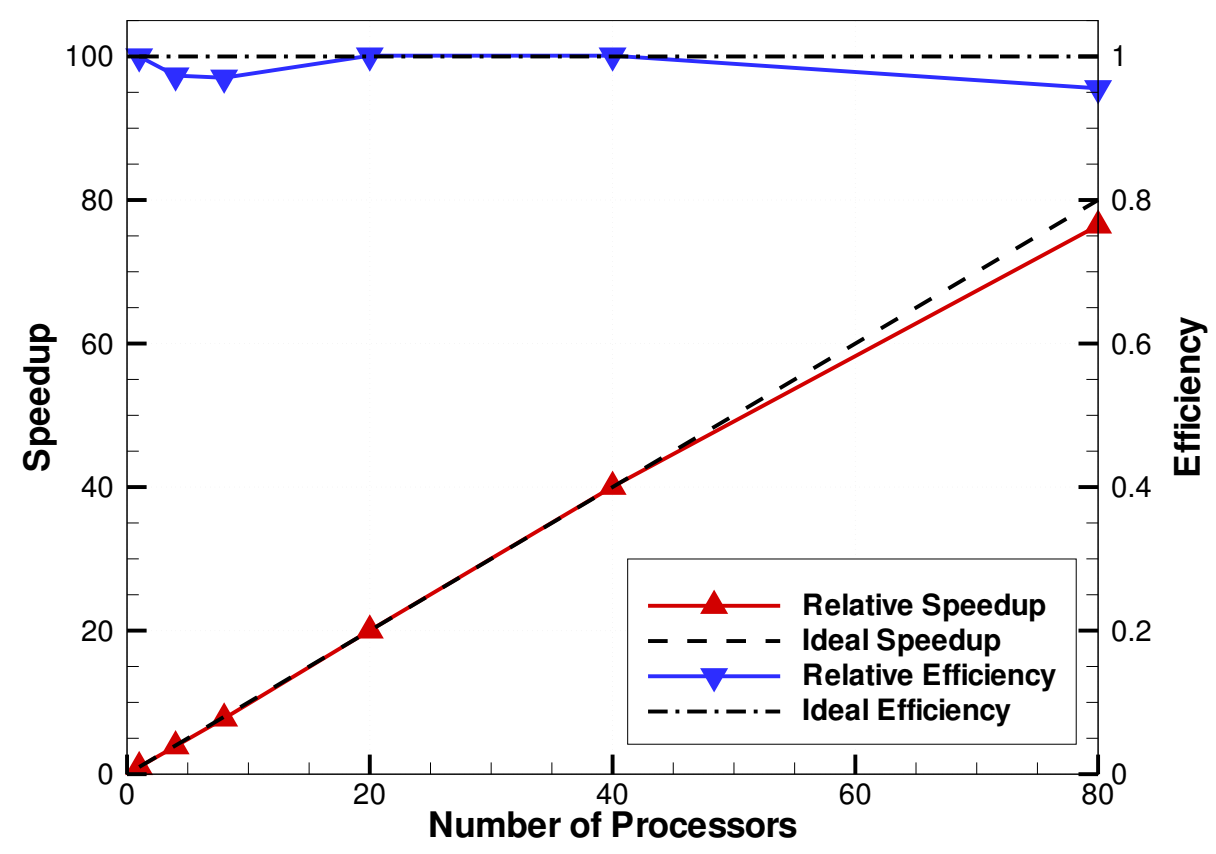

Figure 3. Parallel speedup and efficiency for a problem comprising 64 blocks of $32 \times 64$ cells.

Figure 3. The figure depicts both the relative parallel speed-up, $S_{p}$, given by

$$
S_{p}=\frac{t_{1}}{t_{p}} p
$$

and the relative parallel efficiency, $E_{p}$, given by

$$
E_{p}=\frac{S_{p}}{p}
$$

for a problem comprising 80 blocks of $32 \times 64$ cells $(163,840$ cell computational mesh), as a function of the number of processors, $p$, where $t_{p}$ is the total processor time required to solve the problem using $p$ processors and $t_{1}$ is the processor time required to solve the problem using a single processor. It can be seen that the speed up is linear for up to 80 processors, and even when 80 processors are used, the parallel efficiency remains as high as $96 \%$.

\section{Numerical Results}

Application of the Gaussian solver discussed above to some standard flow problems is now considered for a range of Knudsen numbers. For the determination of the mean free path, the gas is assumed to be comprised of hard spheres for which

$$
\lambda=\frac{16 \mu}{5(2 \pi \rho p)^{\frac{1}{2}}} .
$$

\section{A. Subsonic Planar Couette Flow}

A good first test case to determine the validity of boundary conditions for the Gaussian closure is planar subsonic Couette flow between to oppositely moving infinite plates. $\frac{53}{3}$ Figure 4 shows both the predicted 

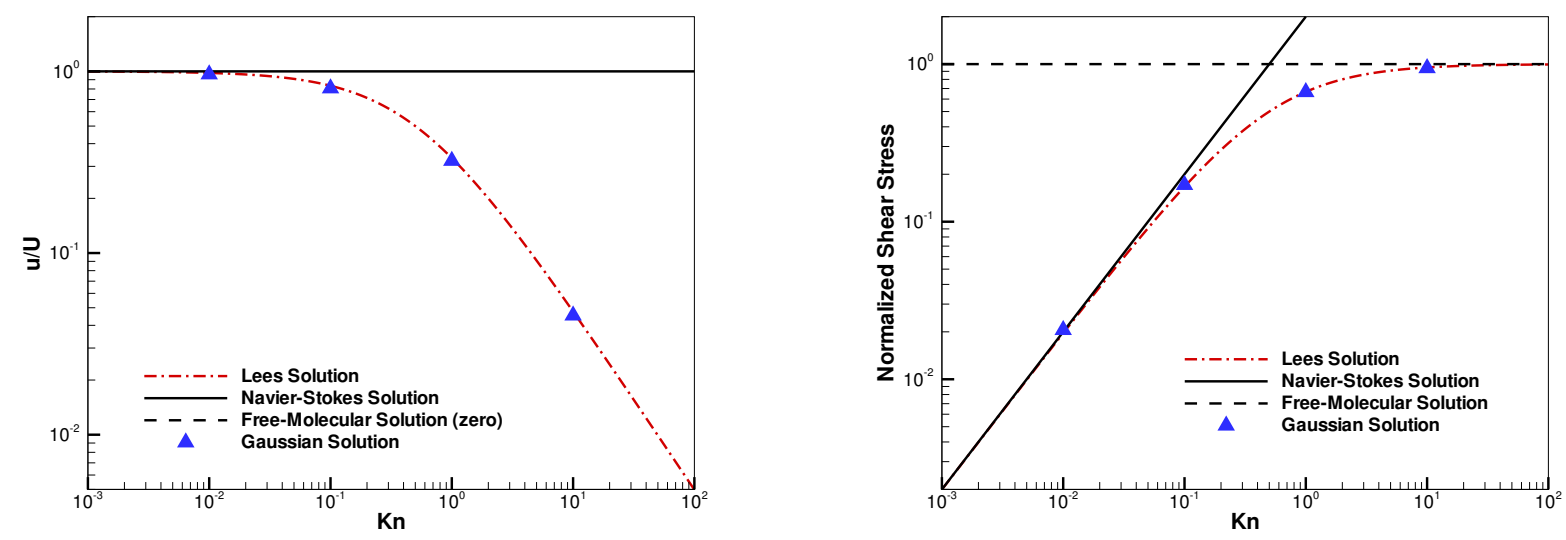

Figure 4. Left: Normalized flow velocity at the wall as a function of Knudsen number. Right: Normalized shear stress as a function of Knudsen number. Both for planar Couette flow between two diffusely reflecting walls.

normalized flow velocity, $u / U$, and normalized shear stress, $\frac{\tau_{x y}}{\rho U \sqrt{\frac{2 k T}{\pi m}}}$ for the case of Couette flow with two plates moving in opposite directions at velocity $U$ and a fluid at temperature $T$ and density $\rho$. The fluid in this case is argon at $288 \mathrm{~K}$ and standard pressure. The two plates move in opposite directions at $30 \mathrm{~m} / \mathrm{s}$. The results show that the Gaussian closure is able to reproduce the correct solutions in both the the continuum and free-molecular flow limits and it effectively transitions from the continuum solution to the free-molecular solution in a manner that is in very good agreement with the approximate analytical solution developed by Lees $[53$

\section{B. Subsonic Flat Plate Boundary Layer Flow}

The application of the Gaussian closure to the prediction of a developing boundary layer over a horizontal flat plate is considered next. Computed Gaussian solutions for air flow over a flat plate with a freestream Mach number of 0.2 are shown in Figure 5. The solution for a low-Knudsen-number continuum flow case $\left(K n=2 \times 10^{-5}\right)$ is shown along with the solution for a higher-Knudsen-number transitional flow case $(K n=0.2)$. The Gaussian solutions are compared to the classical boundary layer flow solution of Blausius. 54 The results for the continuum flow case clearly demonstrate the ability of the Gaussian closure to reproduce the expected incompressible fluid dynamic solution when collisional process are significant. As expected, the velocity slip at the wall is negligible for the low-Knudsen-number case. For the transition regime case, however, the boundary layer becomes thicker and there is now appreciable slip at the solid boundary; these are both expected characteristics of higher Knudsen number flows. The solutions for the flat plate cases were obtained using adaptive mesh refinement. Final mesh resolutions ranged from 42000 to 51000 cells.

\section{Subsonic Flow Past a Circular Cylinder}

There is a reasonable amount of data and theory available in the literature for subsonic steady flow past a circular cylinder. In particular the coefficient of drag, $C_{d}$, is available for continuum, transitional, and free-molecular flow regimes. Figure 6 shows a comparison of experimental data collected by Coudeville et $a l$ with an approximate solution developed by Patterson, 56 and solutions from the Gaussian closure for flow past a cylinder at two different speed ratios, $S$. The speed ratio is the ratio of the bulk speed to the most probable random speed of a particle, it differs from the Mach number by only a constant. The solution due to Patterson is only valid in the region where the Reynolds number is less than 0.5 and where the Knudsen number can be regarded as small (i.e. less than unity). As shown in the figure, this is a very limited range of validity. The comparisons of Figure 6 show that the Gaussian solutions are in very good agreement with the experimental results for the continuum regime and the transition regime. However, as the free-molecular 

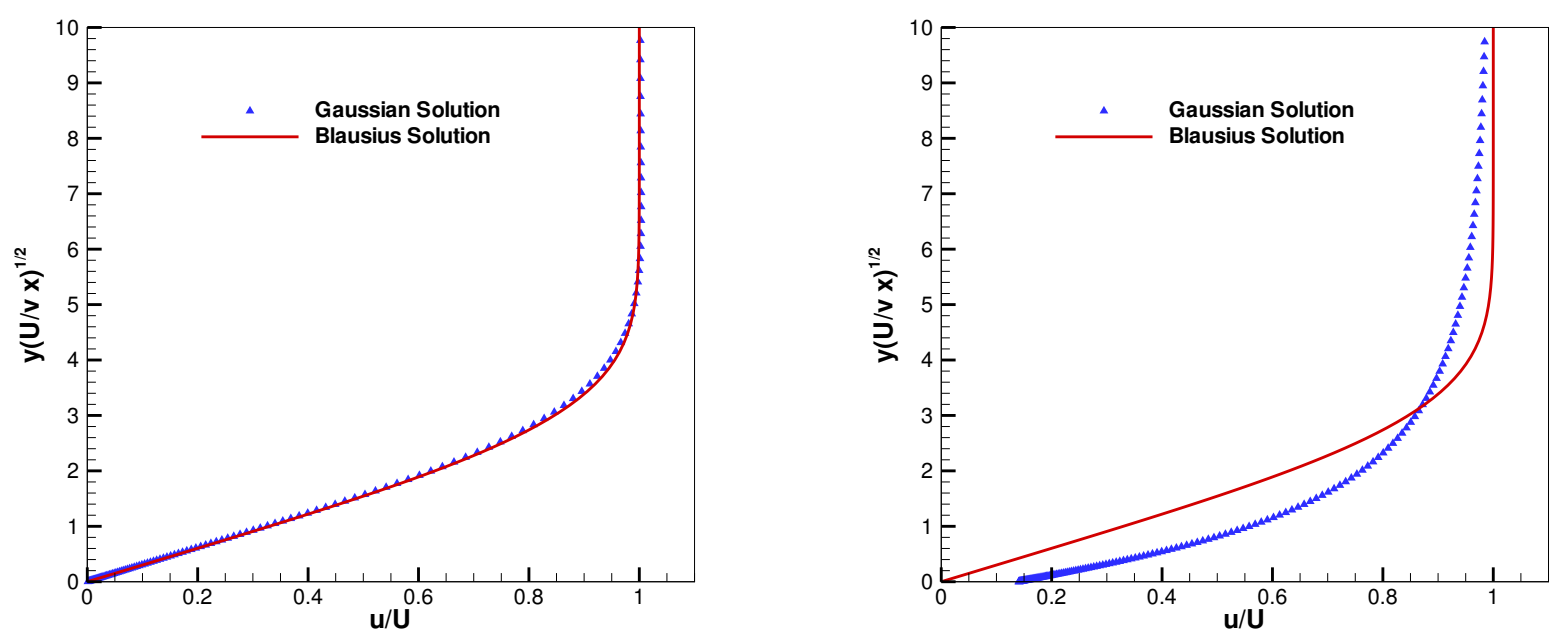

Figure 5. Normalized velocity distribution in the developing boundary layer along a flat plate. Left: Continuum regime; $K n=2 \times 10^{-5}$. Right: Transition regime; $K n=2 \times 10^{-1}$.

regime is approached $(K n>1)$, the numerical predictions of the drag coefficient provided by the Gaussian closure is slightly over-estimated. Gaussian solutions for these cases were obtained on a grid of 10000 cells.

Perhaps a more revealing picture of non-equilibrium effects is provided by the plot of the cylinder drag coefficient as a function of Knudsen number, for a fixed Reynolds number, given in Figure 7 . The figure shows the coefficient of drag for air flow past a cylinder at a Reynolds number of 0.5. Again, the approximate solution of Patterson is shown, as well as the continuum and free-molecular solutions. It can be seen that the Gaussian solution shows good agreement with that of Patterson for $K n<1$; above that range the coefficient of drag is somewhat over-predicted. Gaussian solutions for these cases were obtained using adaptive mesh refinement. Typical final meshes contained between 100000 and 200000 cells.

Finally, a comparison of the flow structure for subsonic flows past a cylinder with a speed ratio $S=0.027$ at two different Knudsen numbers is depicted in Figure 8. Results for $K n=1 \times 10^{-3}$ and $K n=1$ are given. The figures shows that there are marked differences in flow structure of the predicted continuum and non-equilibrium flow solutions. At a Knudsen number of $1 \times 10^{-3}$, the flow is clearly separated and there is a significant region of recirculation downstream of the cylinder. This predicted flow structure is in agreement with experimental observations for flows in this regime. For a Knudsen number of unity, the flow remains attached and there is a greater symmetry between the upstream and downstream solutions. Moreover, the flow is perturbed out to much larger relative distances from the cylinder (i.e., out to larger values of $r / d$ where $r$ is the distance from the centre of the cylinder and $d$ is the cylinder diameter) in the non-equilibrium case.

\section{Transonic Flow Past a NACA0012 Micro-Airfoil}

Lastly, the application of the Gaussian closure to the prediction of transonic steady flow around a NACA0012 micro-airfoil at zero angle of attack has also been considered. For the case presented, the freestream values of the flow Mach number, temperature, and density are $0.8,257 \mathrm{~K}$, and $1.161 \times 10^{-4} \mathrm{Kg} / \mathrm{m}^{3}$, respectively, and the chord length of the airfoil is $0.04 \mathrm{~m}$. These conditions correspond to a Knudsen number of 0.017 . This case was also considered in recent work by Suzuki and van Leer $\frac{16}{16}$ A portion of the body-fitted multi-block AMR grid used for this case is shown in Figure 9. Initially the grid comprises two blocks, each containing 512 cells. After several levels of mesh refinement, the grid comprises 254 blocks and a total of 130,048 cells. It can be seen that the algorithm effectively concentrated cells in areas of high solution gradients near the surface of the airfoil. A plot of the normalized density contours for this case is shown in Figure 10. The predicted results can be compared to the previous computational and experimental results presented by Suzuki and van Leer ${ }^{16}$ Note that an equilibrium result provided by the solution of the Euler equations governing inviscid 


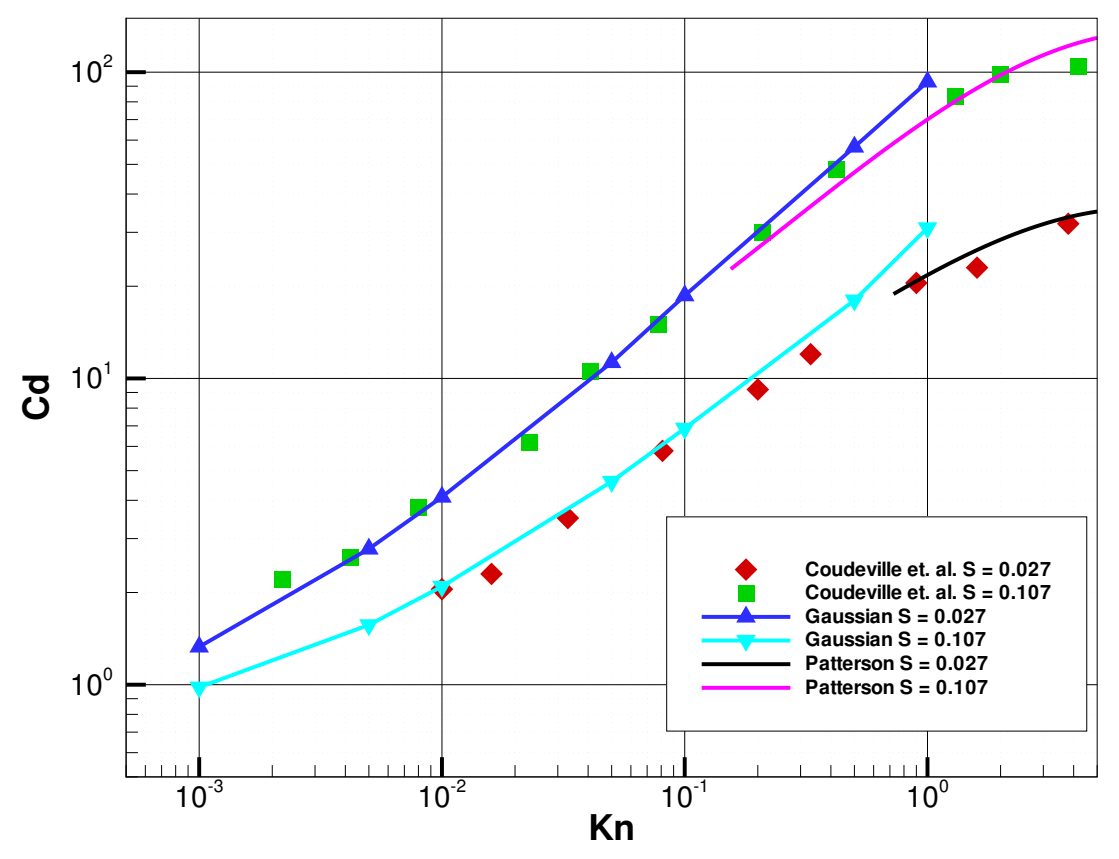

Figure 6. Coefficient of drag for airflow past a circular cylinder at two speed ratios (S): experimental results of Coudeville et al., approximate solution due to Patterson, and Gaussian solution.

flow for this case would have shocks forming on the upper and lower surfaces the airfoil, which are clearly not present in the non-equilibrium solution of the Gaussian closure. The solution of Figure 10 shows fairly good agreement with experimental and other computational results given by Suzuki and van Leer near the leading edge of the airfoil. However, there are some discrepancies in the predicted density field along the length of the upper and lower surfaces of the airfoil which may be attributed to the specification of boundary conditions on the solid surface. Somewhat similar results were obtained by Suzuki and van Leer. Further investigation is required.

\section{Conclusion}

This study has considered the application of the Gaussian closure to micron-scale flow problems. In general, the preliminary numerical results described herein are very encouraging. The closure yields a set of hyperbolic equations that are suitable for solution on irregular meshes, such as those often produced by adaptive mesh refinement. It is also shown that this closure can provide solutions for flows ranging from the continuum regime, through the transition regime and into the high Knudsen number free-molecular flow regime. The lack of heat transfer in this model limits its range of applicability; further research will explore the possibility of an extension to the Gaussian model that will allow for heat transfer.

\section{Acknowledgments}

This research was supported by the Natural Sciences and Engineering Research Council of Canada. Funding for the parallel computing facility used to perform the computations described herein was obtained from the Canadian Foundation for Innovation and Ontario Innovation Trust (CFI Project No. 2169). The authors are very grateful to these funding agencies for this support. The first author would also like to thank the Natural Science and Engineering Research Council of Canada for their generous support of his research through a Canadian Graduate Scholarship. 


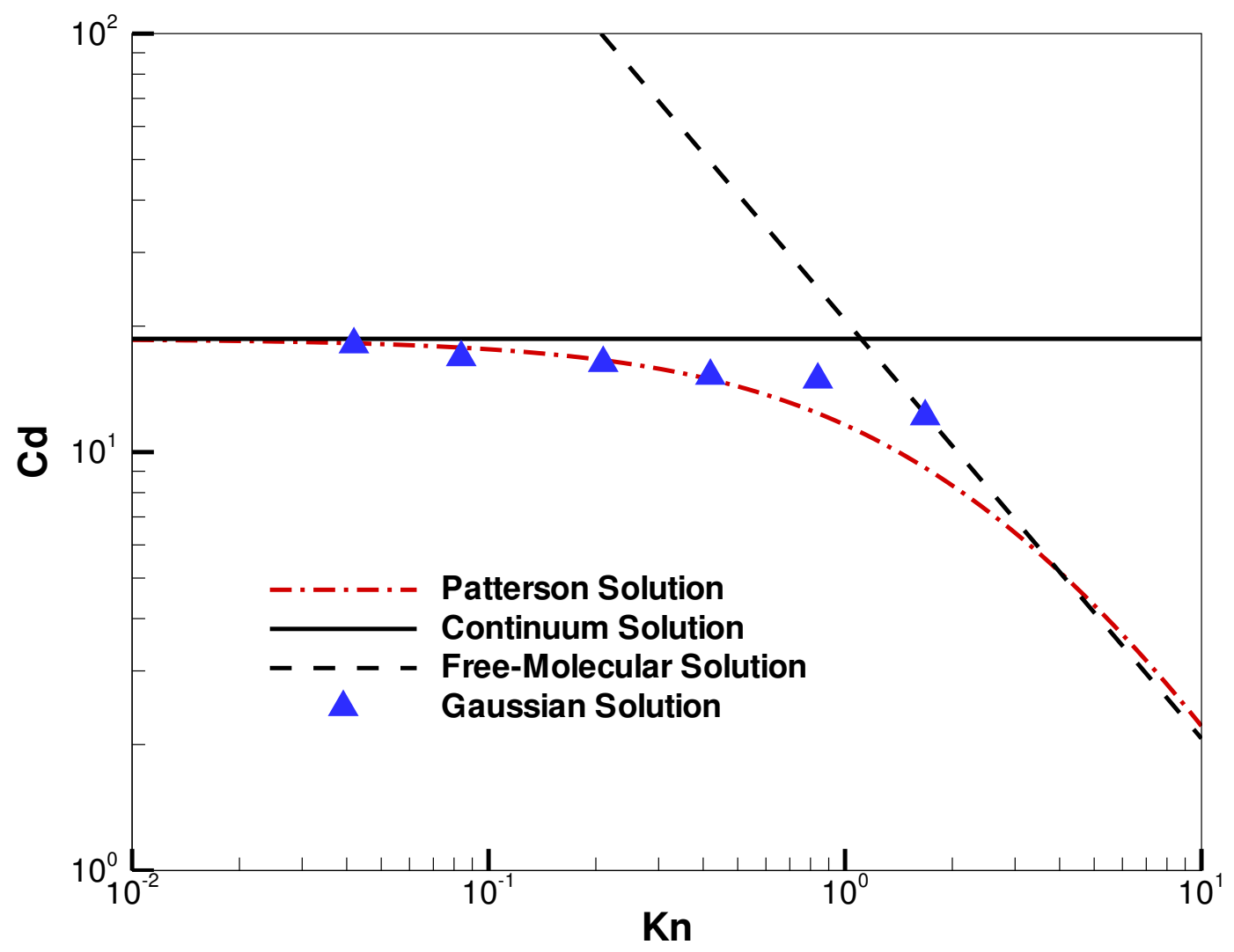

Figure 7. Coefficient of drag for airflow past a circular cylinder at a Reynolds number of 0.5 as a function of Knudsen number
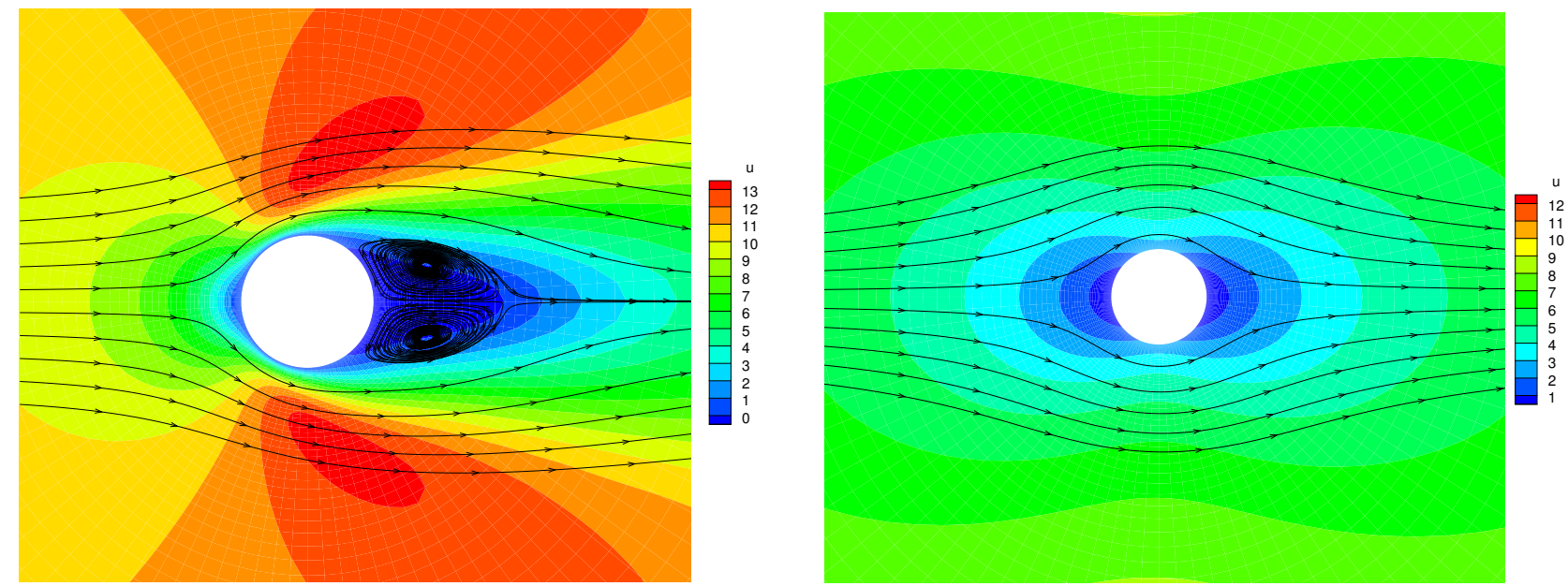

Figure 8. Comparison of $\mathrm{x}$-direction velocity contours for flow past a circular cylinder at a speed ratio $S=0.027$. Left: $K n=1 \times 10^{-3}$. Right: $K n=1$. 

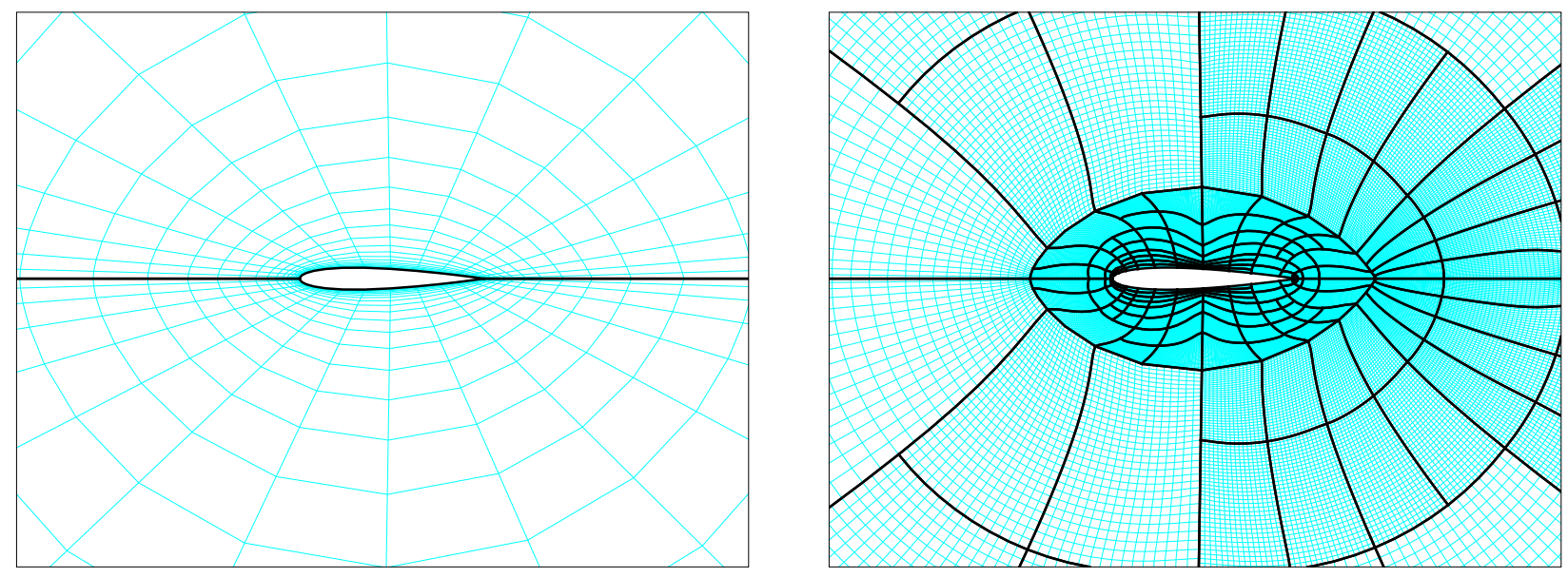

Figure 9. Initial and refined computational AMR mesh used to predict flow past a NACA0012 micro-airfoil.

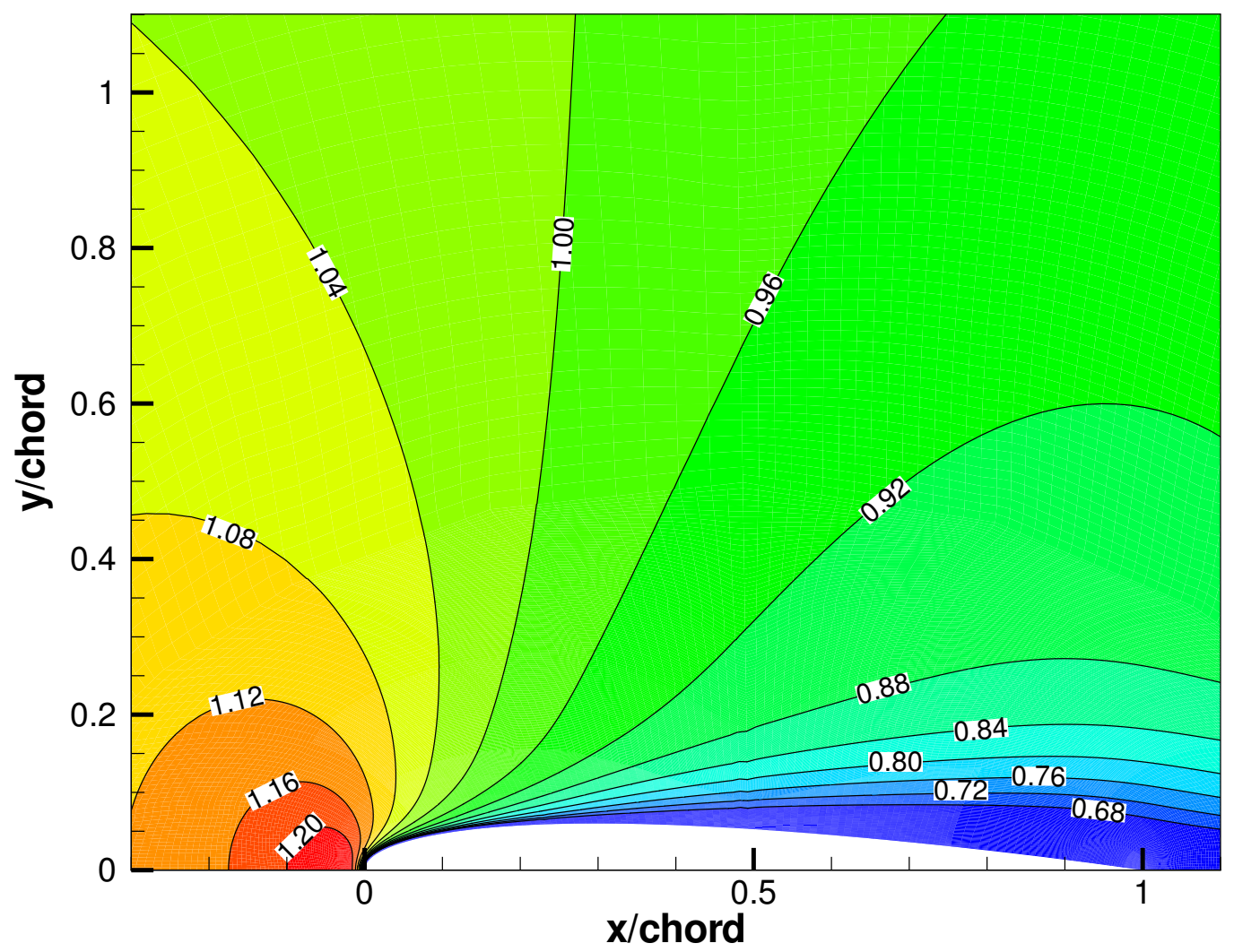

Figure 10. Predicted normalized density contours for Mach 0.8 flow past a NACA0012 micro-airfoil with zero angle of attack. 


\section{References}

47.

${ }^{1}$ O'Connor, L., "MEMS: Mircoelectromechanical Systems," Mechanical Engineering Journal, Vol. 114, No. 2, 1992, pp. 40-

${ }^{2}$ Meyyappan, M., editor, Computational Modeling in Semiconductor Processing, Artech House, Boston, 1995.

${ }^{3}$ Beskok, A. and Karniadakis, G. E., "Simulation of Heat and Momentum Transfer in Complex Microgeometries," Journal of Thermophysics and Heat Transfer, Vol. 8, No. 4, 1994, pp. 647-655.

${ }^{4}$ Harley, J. C., Huang, Y., Bau, H. B., and Zemel, J. N., "Gas-Flow in Micro-Channels," Journal of Fluid Mechanics, Vol. 284, 1995, pp. 257-274.

${ }^{5}$ Oh, C. K., Oran, E. S., and Cybyk, B. Z., "Microchannel Flow Computed with the DSMC-MLG," Paper 95-2090, AIAA, June 1995.

${ }^{6}$ Piekos, E. S. and Breuer, K. S., "DSMC Modeling of Micromechanical Devices," Paper 95-2089, AIAA, June 1995.

${ }^{7}$ Bird, G. A., Molecular Gas Dynamics and the Direct Simulation of Gas Flows, Clarendon Press, Oxford, 1994.

${ }^{8}$ Breuer, K. S., Piekos, E. S., and Gonzales, D. A., "DSMC Simulations of Continuum Flows," Paper 95-2088, AIAA, June 1995.

${ }^{9}$ Levermore, C. D., "Moment Closure Hierarchies for Kinetic Theories," Journal of Statistical Physics, Vol. 83, 1996, pp. 1021-1065.

${ }^{10}$ Groth, C. P. T., Gombosi, T. I., Roe, P. L., and Brown, S. L., "Gaussian-Based Moment-Method Closures for the Solution of the Boltzmann Equation," Proceedings of the Fifth International Conference on Hyperbolic Problems - Theory, Numerics, Applications, University of New York at Stony Brook, Stony Brook, New York, U.S.A., June 13-17, 1994, edited by J. Glimm, M. J. Graham, J. W. Grove, and B. J. Plohr, World Scientific, New Jersey, 1996, pp. 339-346.

${ }^{11}$ Groth, C. P. T., Roe, P. L., Gombosi, T. I., and Brown, S. L., "On the Nonstationary Wave Structure of a 35-Moment Closure for Rarefied Gas Dynamics," Paper 95-2312, AIAA, June 1995.

${ }^{12}$ Brown, S. L., Roe, P. L., and Groth, C. P. T., "Numerical Solution of a 10-Moment Model for Nonequilibrium Gasdynamics," Paper 95-1677, AIAA, June 1995.

${ }^{13}$ Brown, S. L., Approximate Riemann Solvers for Moment Models of Dilute Gases, Ph.D. thesis, University of Michigan, 1996.

${ }^{14}$ Levermore, C. D. and Morokoff, W. J., "The Gaussian Moment Closure for Gas Dynamics," SIAM Journal on Applied Mathematics, Vol. 59, No. 1, 1996, pp. 72-96.

${ }^{15}$ Hittinger, J. A., Foundations for the Generalization of the Godunov Method to Hyperbolic Systems with Stiff Relaxation Source Terms, Ph.D. thesis, University of Michigan, 2000.

${ }^{16}$ Suzuki, Y. and van Leer, B., "Application of the 10-moment model to MEMS flows," Paper 2005-1398, AIAA, January 2005.

${ }^{17} \mathrm{Grad}, \mathrm{H} .$, "On the Kinetic Theory of Rarefied Gases," Communications on Pure and Applied Mathematics, Vol. 2, 1949, pp. 331-407.

${ }^{18}$ Chapman, S., "On the Kinetic Theory of a Gas. Part II. - A Composite Monoatomic Gas: Diffusion, Viscosity, and Thermal Conduction," Philosophical Transactions of the Royal Society of London A, Vol. 217, 1916, pp. 115-116.

${ }^{19}$ Enskog, D., Kinetishe Theorie der Vorgänge in Massing Verdumten Gasen, Ph.D. thesis, University of Upsala, 1917.

${ }^{20}$ Burnett, D., "The Distribution of Velocities in a Slightly Non-Uniform Gas," Proceedings of the London Mathematics Society, Vol. 39, 1935, pp. 385-430.

${ }^{21}$ Godunov, S. K., "Finite-Difference Method for Numerical Computations of Discontinuous Solutions of the Equations of Fluid Dynamics," Matematicheskii Sbornik, Vol. 47, 1959, pp. 271-306.

${ }^{22}$ van Leer, B., "Towards the Ultimate Conservative Difference Scheme. V. A Second-Order Sequel to Godunov's Method," Journal of Computational Physics, Vol. 32, 1979, pp. 101-136.

${ }^{23}$ Harten, A., "High Resolution Schemes for Hyperbolic Conservation Laws," Journal of Computational Physics, Vol. 49, 1983, pp. 357-393.

${ }^{24}$ Colella, P. and Woodward, P. R., "The Piecewise Parabolic Method (PPM) for Gas-Dynamical Simulations," Journal of Computational Physics, Vol. 54, 1984, pp. 174-210.

${ }^{25}$ Yee, H. C., "Construction of Explicit and Implicit Symmetric TVD Schemes and Their Applications," Journal of Computational Physics, Vol. 68, 1987, pp. 151-179.

${ }^{26}$ Berger, M. J. and Colella, P., "Local Adaptive Mesh Refinement for Shock Hydrodynamics," Journal of Computational Physics, Vol. 82, 1989, pp. 67-84

${ }^{27}$ De Zeeuw, D. and Powell, K. G., "An Adaptively Refined Cartesian Mesh Solver for the Euler Equations," Journal of Computational Physics, Vol. 104, 1993, pp. 56-68.

${ }^{28}$ Aftomis, M. J., Berger, M. J., and Melton, J. E., "Robust and Efficient Cartesian Mesh Generation for Component-Base Geometry," AIAA Journal, Vol. 36, No. 6, 1998, pp. 952-960.

${ }^{29}$ Quirk, J. J. and Hanebutte, U. R., "A Parallel Adaptive Mesh Refinement Algorithm," Report 93-63, ICASE, August 1993.

${ }^{30}$ Groth, C. P. T., Zeeuw, D. L. D., Powell, K. G., Gombosi, T. I., and Stout, Q. F., "A Parallel Solution-Adaptive Scheme for Ideal Magnetohydrodynamics," Paper 99-3273, AIAA, June 1999.

${ }^{31}$ Aftomis, M. J., Berger, M. J., and Murman, S. M., "Applications of Space-Filling Curves to Cartesian Methods for CFD," Paper 2004-1232, AIAA, January 2004.

${ }^{32}$ Chapman, S. and Cowling, T. G., The Mathematical Theory of Non-Uniform Gases, Cambridge University Press, Cambridge, 1960.

${ }^{33}$ Burgers, J. M., Flow Equations for Composite Gases, Academic Press, New York, 1969

${ }^{34}$ Gombosi, T. I., Gaskinetic Theory, Cambridge University Press, Cambridge, 1994. 
${ }^{35}$ Bhatnagar, P. L., Gross, E. P., and Krook, M., "A Model for Collision Processes in Gases. I. Small Amplitude Processes in Charged and Neutral One-Component Systems," Physical Review, Vol. 94, No. 3, 1954, pp. 511-525.

${ }^{36}$ Maxwell, J. C., "On the Dynamical Theory of Gases," Philosophical Transactions of the Royal Society of London, Vol. 157, 1867, pp. 49-88.

${ }^{37}$ Hertweck, F., "Allgemeine 13-Momenten-Näherung zur Fokker-Planck-Gleichung eines Plasmas," Zeitschrift für Naturforschung, Vol. 20a, 1965, pp. 1243-1255.

${ }^{38}$ Oraevskii, V., Chodura, R., and Feneberg, W., "Hydrodynamic Equations for Plasmas in Strong Magnetic Fields - I Collisionless Approximation," Plasma Physics, Vol. 10, 1968, pp. 819-828.

${ }^{39}$ Holway, L. H., Approximation Procedures for Kinetic Theory, Ph.D. thesis, Harvard University, 1963.

${ }^{40}$ Holway, L. H., "Kinetic Theory of Shock Structure Using an Ellipsoidal Distribution Function," Rarefied Gas Dynamics, edited by J. H. de Leeuw, Vol. I, Academic Press, New York, 1966, pp. 193-215.

${ }^{41}$ Holway, L. H., "The Effect of Collisional Models upon Shock Wave Structure," Rarefied Gas Dynamics, edited by C. L. Brundin, Vol. I, Academic Press, New York, 1967, pp. 759-784.

${ }^{42}$ Chodura, R. and Pohl, F., "Hydrodynamic Equations for Plasmas in Strong Magnetic Fields - II Transport Equations Including Collisions," Plasma Physics, Vol. 13, 1971, pp. 645-658.

${ }^{43}$ Demars, H. G. and Schunk, R. W., "Transport Equations for Multispecies Plasmas Based on Individual Bi-Maxwellian Distributions," Journal of Physics D: Applied Physics, Vol. 12, 1979, pp. 1051-1077.

${ }^{44}$ Roe, P. L., "Approximate Riemann Solvers, Parameter Vectors, and Difference Schemes," Journal of Computational Physics, Vol. 43, 1981, pp. 357-372.

${ }^{45}$ Barth, T. J., "Recent Developments in High Order K-Exact Reconstruction on Unstructured Meshes," Paper 93-0668, AIAA, January 1993

${ }^{46}$ Venkatakrishnan, V., "On the Accuracy of Limiters and Convergence to Steady State Solutions," Paper 93-0880, AIAA, January 1993.

${ }^{47}$ Einfeldt, B., "On Godunov-Type Methods for Gas Dynamics," SIAM Journal on Numerical Analysis, Vol. 25, 1988, pp. $294-318$

${ }^{48}$ Sachdev, J. S., Groth, C. P. T., and Gottlieb, J. J., "A Parallel Solution-Adaptive Scheme for Predicting Multi-Phase Core Flows in Solid Propellant Rocket Motors," International Journal of Computational Fluid Dynamics, Vol. 19, No. 2, 2005, pp. $157-175$.

${ }^{49}$ Berger, M. J. and Saltzman, J. S., "AMR on the CM-2," Applied Numerical Mathematics, Vol. 14, 1994, pp. $239-253$.

${ }^{50}$ Quirk, J. J., An Adaptive Grid Algorithm for Computational Shock Hydrodynamics, Ph.D. thesis, Cranfield Institute of Technology, January 1991.

${ }^{51}$ Davis, R. L. and Dannenhoffer, J. F., "Decomposition and Parallelization Strategies for Adaptive Grid-Embedding Techniques," International Journal of Computational Fluid Dynamics, Vol. 1, 1993, pp. 79-93.

${ }^{52}$ Sun, M. and Takayama, K., "Conservative Smoothing on an Adaptive Quadrilateral Grid," Journal of Computational Physics, Vol. 150, 1999, pp. 143-180.

${ }^{53}$ Vincenti, W. G. and Kruger, C. H., Introduction to Physical Gas Dynamics, R. E. Krieger Publishing, Huntington, NY, 1975.

${ }^{54}$ Schlichting, H., Boundary-Layer Theory, McGraw-Hill, Toronto, 7th ed., 1979.

${ }^{55}$ H. Coudeville, P. T. and Brun, E., "Drag Measurements in Slip and Transition Flow," Proceedings of the Fourth International Symposium on Rarefied Gas Dynamics, Academic Press, New York, 1965.

${ }^{56}$ Patterson, G. N., Introduction to the Kinetic Theory of Gas Flows, University of Toronto Press, Toronto, 1961. 